\title{
ON SPACES OF OPERATORS WHOSE DUALS ARE ISOMETRIC TO $L^{1}(\mu)$
}

\author{
T. S. S. R. K. RAO
}

Abstract. Following the well-known classification scheme of function spaces whose duals are isometric to $L^{1}(\mu)$, due to Lindenstrauss, Wulbert and Olsen, in this paper we study the geometric properties of space of compact operators $\mathscr{K}(E, F)$ under the assumption that it is in one of the classes described by Lindenstrauss, Wulbert and Olsen.

Mathematics subject classification (2000): 46E40, 46B20.

Keywords and phrases: $L^{1}$-preduals, injective, projective tensor products.

\section{REFERENCES}

[1] M. CAmbern And P. GReim, The dual of a space of vector measures, Math. Z., 180 (1982) 373-378.

[2] J. Diestel And J. J. Uhl, Vector measures. With a foreword by B. J. Pettis, Mathematical Surveys, No. 15. American Mathematical Society, Providence, R.I., 1977. xiii+322 pp.

[3] P. Harmand, D. Werner And W. Werner, M-ideals in Banach spaces and Banach algebras, Lecture Notes in Mathematics, 1547. Springer-Verlag, Berlin, 1993. viii+387 pp.

[4] H. E. LACEY, The isometric theory of classical Banach spaces, Die Grundlehren der mathematischen Wissenschaften, Band 208. Springer-Verlag, New York-Heidelberg, 1974. x+270 pp.

[5] J. Lindenstrauss AND D. E. WulBert, On the classification of the Banach spaces whose duals are $L_{1}$ spaces, J. Functional Analysis, 4 (1969) 332-349.

[6] A. Lima AND G. H. Olsen, Extreme points in duals of complex operator spaces, Proc. Amer. Math. Soc., 94 (1985) 437-440.

[7] G. H. OLSEn, On the classification of complex Lindenstrauss spaces, Math. Scand., 35 (1974) 237258.

[8] T. S. S. R. K. RAO, A. K. RoY AND K. Sundaresan, Intersection properties of balls in tensor products of some Banach spaces, Math. Scand., 65 (1989) 103-118.

[9] T. S. S. R. K. RAO, Spaces of operators as continuous function spaces, Extracta Mathematicae, 12 (1997) 251-253.

[10] T. S. S. R. K. RAO, A note on a paper of Ercan and Önal, Tiwanese Journal of Mathematics, to appear, 2009.

[11] W. RUESS, Duality and geometry of spaces of compact operators, Functional analysis: surveys and recent results, III (Paderborn, 1983) 59-78, North-Holland Math. Stud., 90, North-Holland, Amsterdam, 1984. 